INPLASY

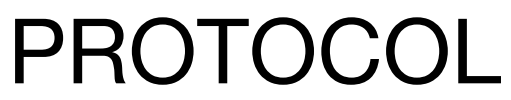

To cite: Zhou et al. Effect of

Sancai Powder on Glacemic

Variability of Type 1 Diabetes in

China. Inplasy protocol

202050035. doi:

10.37766/inplasy2020.5.0035

Received: 09 May 2020

Published: 09 May 2020

Corresponding author:

Dong Qi Zhou

973610074@qq.com

Author Affiliation:

Hospital of Chengdu University

of Traditional Chin

Support: CYW2019079 and No. 2019YFS0085

Review Stage at time of this submission: The review has not yet started.

Conflicts of interest:

The authors report no conflicts of interest in this work.

\section{Effect of Sancai Powder on Glacemic Variability of Type 1 Diabetes in China}

\author{
Zhou, DQ'; Zhang, L²; Chen, R³ Yu, W4; Zhang, P5; Chen, Q6.
}

Review question / Objective: 1.Efficacy and safety of Sancai powder on Chinese patients with type 1 diabetes. 2. Whether Sancai powder can effectively reduce the blood sugar variability of patients with type 1 diabetes in China.

Condition being studied: T1DM is a chronic, immunemediated disease characterized by the destruction of insulin producing cells and persistent hyperglycemia. At present, the drugs for T1DM can reduce blood glucose rapidly and effectively, but there are risks of hypoglycemia, large fluctuation of blood glucose and chronic complications. Related research found that compared with continuous hyperglycemia, blood glucose fluctuations are more harmful to the chronic complications of diabetes. Blood glucose variation is closely related to the occurrence and development of chronic complications of diabetes. Sancai powder(SC)is made on the basis of three ancient Chinese medicine formulas, which has the effect of lowering blood glucose. There have been reports on the clinical study of SC in the treatment of diabetic patients, but there is no systematic evaluation of SC in the treatment of type 1 diabetes, so it is necessary to summarize and evaluate the existing evidence.

INPLASY registration number: This protocol was registered with the International Platform of Registered Systematic Review and Meta-Analysis Protocols (INPLASY) on 09 May 2020 and was last updated on 09 May 2020 (registration number INPLASY202050035).

\section{INTRODUCTION}

Review question / Objective: 1.Efficacy and safety of Sancai powder on Chinese patients with type 1 diabetes. 2. Whether Sancai powder can effectively reduce the blood sugar variability of patients with type 1 diabetes in China.

Condition being studied: T1DM is a chronic, immune-mediated disease characterized by the destruction of insulin producing cells and persistent hyperglycemia. At 
present, the drugs for T1DM can reduce blood glucose rapidly and effectively, but there are risks of hypoglycemia, large fluctuation of blood glucose and chronic complications. Related research found that compared with continuous hyperglycemia, blood glucose fluctuations are more harmful to the chronic complications of diabetes. Blood glucose variation is closely related to the occurrence and development of chronic complications of diabetes. Sancai powder(SC)is made on the basis of three ancient Chinese medicine formulas, which has the effect of lowering blood glucose. There have been reports on the clinical study of SC in the treatment of diabetic patients, but there is no systematic evaluation of $S C$ in the treatment of type 1 diabetes, so it is necessary to summarize and evaluate the existing evidence.

\section{METHODS}

Participant or population: Patients with Type 1 Diabetes in China.

Intervention: San cai powder.

Comparator: Placebo or conventional treatments.Conventional treatments include oral hypoglycemic medications and insulin injections. Of course, insulin pumps are among the routine treatments.

Study designs to be included: Those randomized controlled trials that treated T1DM with SC in combination with placebo or conventional treatments will be included.

Eligibility criteria: Randomized controlled trials that treated T1DM with SC in combination with placebo or conventional treatments.

Information sources: 4 Chinese databases, including Articles knowledge service platform, VIP information resource integration Service platform (cqvip), the China National Knowledge Infrastructure (CNKI) database,China Biomedical CD (Sino Med), as well as 3 English databases, including Embase,PubMed, Cochrane Library Controlled Trials Register,Chinese and English restricted languages. In addition, to identify unpublished studies or other relevant documents, we will also search Google Scholar, Baidu scholars.Last but not least, the Chinese Clinical Trials Registry (ChiCTR) and ClinicalTrials.gov will also be searched.

Main outcome(s): Standard deviation of blood glucose level (SDBG), coefficient of variation (CV).

Additional outcome(s): 1. MBG, PPG, hypoglycemia index,glycated hemoglobin. 2. Total effective rate; The judgement of effectiveness takes into account the improvement of examination indicators and the relief of clinical symptoms. 3.Hyperglycemia or hypoglycemia that may occur during the study. 4. Have any adverse effects of medication throughout the process.

Quality assessment / Risk of bias analysis: The two reviewers will use a mature and reliable way to assess the risk of bias in the study: the Cochrane Collaboration tool. In this tool, seven items were used to evaluate the risk of experimental bias: random sequence generation (selection bias), assignment concealment (selection bias), blindness of participants and personnel (performance bias), blind (detection) bias of result evaluation, incomplete result data (loss bias), selective reporting (reporting bias), and other biases. Each project is divided into "low risk", "high risk" or "unclear risk".

Strategy of data synthesis: For continuous variables, the effect is expressed as mean difference (MD) and $95 \% \mathrm{Cl}$. For binary variables, the impact will be expressed as a risk ratio (RR) and a $95 \%$ confidence interval $(\mathrm{Cl})$ and will be calculated using the mantel-Haenszel (MH) method. The heterogeneity of the data will be studied by Cochrane $\times 2$ and 12 tests. [27] When p 50\%, statistical heterogeneity is considered important. If $\mathrm{P}>\mathbf{0 . 0 5}$ and $12<50 \%$, the included studies are homogeneous and the difference between the two is negligible. If there is significant heterogeneity, the random effect model is used to aggregate 
the data; if there is no significant heterogeneity, the fixed effect model is used. If a large amount of heterogeneity does not allow quantitative synthesis, the results will be presented in the form of tables and charts.

Subgroup analysis: When there is great heterogeneity between studies, we will conduct subgroup analysis and metaregression to explore heterogeneity. The patient's condition at baseline, the type of medication used during treatment, and the duration of intervention are the three hypotheses we established for the subgroup analysis. [28]] We will perform subgroup analysis based on these subgroup hypotheses. Then, we will evaluate the credibility of the subgroup analysis according to reliable subgroup analysis guidelines. [29] If there is enough research, then meta-regression will be conducted to further explore the source of heterogeneity.

Sensibility analysis: The purpose of conducting sensitivity analysis is to ensure the stability of the research results. Each study included in the analysis will be excluded one by one, and then re-analyze and summarize the data, and compare the difference between the regained effect and the original effect. In this way, the impact of a single study on the overall results and whether the results are reliable will be assessed.

Language: Chinese and English.

Country(ies) involved: China.

Keywords: saicai powder, Glacemic Variability, Type 1 Diabetes, traditional Chinese medicine.

Contributions of each author:

Author 1 - Dongqi Zhou.

Author 2 - Li Zhang.

Author 3 - Rumeng Chen.

Author 4 - Weiwei Yu.

Author 5 - Peishuai Zhang.

Author 6 - Qiu Chen. 